Applied Physics Report 94-40

\title{
Flicker Noise Induced by Dynamic Impurities in a Quantum Point Contact
}

\author{
J. P. Hessling* \\ Department of Applied Physics, Chalmers University of Technology and Göteborg University, \\ S-412 96, Göteborg, Sweden \\ Yu. M. Galperin \\ Department of Physics, University of Oslo, P.O. Box 1048 Blindern, N 0316 Oslo 3, Norway, \\ and A.F. Ioffe Physico-Technical Institute, 194021 St. Petersburg, Russia
}

December 7, 2017

\begin{abstract}
We calculate low-frequency noise (LFN) in a quantum point contact (QPC) which is electrostatically defined in a 2D electron gas of a GaAs-AlGaAs heterostructure. The conventional source of LFN in such systems are scattering potentials fluctuating in time acting upon injected electrons. One can discriminate between potentials of different origin - noise may be caused by the externally applied gate- and source-drain voltages, the motion of defects with internal degrees of freedom close to the channel, electrons hopping between localized states in the doped region, etc. In the present study we propose a model of LFN based upon the assumption that there are many dynamic defects in the surrounding of a QPC. A general expression for the
\end{abstract}

\footnotetext{
*Electronic address: hessling@fy.chalmers.se
} 
time-dependent current-current correlation function is derived and applied to a QPC with quantized conductance. It is shown that the level of LFN is significantly different at and between the steps in a plot of the conductance vs. gate voltage. On the plateaus, the level of noise is found to be low and strongly model-dependent. At the steps, LFN is much larger and only weakly model-dependent. As long as the system is biased to be at a fixed position relative the conductance step, we find that the level of noise is independent of the number of conducting modes. From numerical calculations we conclude that the level of noise approximately obeys a power law as a function of frequency for frequencies larger than a threshold. At the steps for frequencies larger than the minimal transition rate for the dynamical impurities, we have $S(\omega) \propto 1 / \omega^{0.85}$. We are convinced that noise measurements will play a crucial role in the course of investigating the effect of the environment in QPCs. PACS numbers: 72.20.-i, 72.70.+m

\section{INTRODUCTION}

Electron transport through ballistic quantum point contacts (QPC), shown in Fig. 1, has been extensively studied during the last decade (for a review see Ref. [1]). One of the most interesting properties of these systems is the quantization [2] of the conductance as a function of the applied gate voltage (for a review see Ref. [1] and references therein). In addition, small oscillations in the $I-V_{s d}$ curves have been found. The quantum channel is assumed to behave as a wave guide where the number of transverse modes is dependent on the gate voltage $V_{g}$ as well as on the source-drain voltage $V_{s d}$. According to the present understanding (see e.g. Ref. [1]), the steps in the conductance when measured vs. gate voltage are due to switching of the effective number of transverse modes in the channel. The small-scale oscillations in the $I-V_{s d^{-}}$curves are also believed to be caused by this kind of switching [5] 7 . 
Low frequency noise in QPCs may originate from time-dependent internal as well as external parameters. Fluctuations in the potentials applied to the electrodes are examples of external sources of noise. Internal noise can for instance be generated by the motion of impurities within the contact region, or by a time-dependent rearrangement of charge in the doped region of the structure.

Here we focus on noise of internal origin which is intrinsic for any realistic structure. It is well established that there is some disorder in the vicinity of small devices even if they are of high quality. In any disordered system, defects with internal degrees of freedom are present which may change their position due to interactions with a thermal bath. There will hence be a time-dependent impurity potential. These defects usually switch between two states, leading to a telegraph-like noise. Dynamical defects of this type were observed by several authors in the surrounding of even very high quality classical point contacts [8 13]. The role of the defects with internal degrees of freedom in the surrounding of point contacts has been extensively discussed in the literature (for a review see Ref. [14] and references therein). Recently, new features have been studied [15 17] which are due to the Kondo effect in scattering by two-level impurities.

It is quite reasonable to believe that such defects, so-called elementary fluctuators (EFs), also exist in the surrounding of ballistic quantum point contacts. There are experimental results for QPCs which can be explained by the presence of defects with internal degrees of freedom. In particular, we have made an attempt [18] to find theoretical support for experimentally observed correlation between telegraph-like noise and current in QPCs 19]. An oscillating response to small source-drain voltages was found [20,21].

The microscopic structure of the EFs is not yet completely clear. One of the proposed explanations for the presence of two-state defects is disorder-induced soft atomic vibrations. For low excitation energies the vibrations are strongly anharmonic and can be described as an atom or group of atoms moving in an effective double-well potential. Such entities are known as two-level tunneling systems (TLS) [22,23]. These are responsible for the low temperature properties of glassy materials. The generalization of the TLS model for higher 
excitation energies was worked out in Ref. [24]. Dynamical defects produce electric fields, slowly varying in time. The fields will scatter conduction electrons, thereby creating a sort of 'noisy environment'. Another possibility for having fluctuations in the scattering potential is electrons hopping between adjacent impurity centers in the doped region.

Our aim is to analyze low-frequency noise (LFN) in the current, caused by a 'noisy environment' of a QPC. In this study we restrict ourselves to systems with a wide spacer between the doped region and the 2DEG where the quantum channel is defined, see Fig. 11. If the spacer is wide enough (which is the case for high-mobility structures), the correlation length of the impurity potential may exceed the size of the QPC. Hence, the impurities in the doped region will contribute to the smooth static potential felt by the electrons in the quantum channel. A time-dependent rearrangement of the charge in the doped region leads to almost homogeneous fluctuations of the potential in space even if the voltages between the gate electrodes are kept constant. Effectively, these fluctuations manifest themselves as variations in time of the effective gate voltage.

At the plateaus in conductance such fluctuations cannot change the current considerably. However, close to the steps transverse modes may be turned on or off. As a result, the level of LFN should increase significantly in the vicinities of the conductance steps.

The conductance of ballistic channels is a strongly non-linear property. Hence one can expect to find rather unusual and informative properties of LFN of such contacts. Experimental studies may serve as tools for extracting the principal interaction mechanisms between electrons in QPCs and their environment. Studies of this kind may also allow us to draw conclusions about the spatial distribution as well as the intrinsic dynamics of the impurities.

This article is organized as follows. In Section \, the current through an adiabatic contact is discussed which provides us with the starting point of our derivation of the level of noise. The model for low-frequency noise (LFN) in a QPC is formulated in Section III]. In this model, LFN is caused by EFs distributed randomly in the vicinity of a QPC.

General analytical expressions for low-frequency noise as well as results from numerical 
calculations, will be given in Section III. In Section IV we discuss our main results and draw conclusions. We have also submitted a derivation in Appendix A explaining why, under some circumstances, noise is independent of the number of propagating modes.

\section{MODEL}

The system under study consists of an adiabatically smooth channel [25] connecting two equilibrium reservoirs. It is formed in a $2 \mathrm{D}$ electron gas by the split gate technique. The gates are assumed to provide a hard wall potential in the transverse direction $(y)$, slowly (adiabatically) varying along the channel $(x)$. Accordingly, the WKB approximation for the electron wave function is applicable. This wave function can be written as 25]

$$
\Psi(x, y, E)=\sum_{n, \pm} a_{n}^{ \pm} \chi_{n}^{ \pm}(x, y, E)
$$

where

$$
\begin{aligned}
\chi_{n}^{ \pm}(x, y, E) & =\sqrt{k_{n, \|}(E, \mp \infty) / k_{n, \|}(E, x)} \phi_{n, x}(y) \\
& \times \exp \left[i \int_{\mp \infty}^{x} d x^{\prime} k_{n, \|}\left(E, x^{\prime}\right)\right] \\
k_{n, \|}(E, x) & =k_{\mathrm{F}} \sqrt{\varepsilon-\varepsilon_{n, \perp}-\Upsilon(x, t)} .
\end{aligned}
$$

Here $E \equiv \varepsilon E_{\mathrm{F}}$ is the total energy of the electron $-E_{\mathrm{F}}$ being the Fermi energy in the leads at zero bias voltage, $V_{s d}=0$ - while $k_{\mathrm{F}}=\left(2 m E_{\mathrm{F}} / \hbar^{2}\right)^{1 / 2}$ is the Fermi wave vector and $k_{n, \|}(E, x)$ the longitudinal wave vector along the channel. The transverse part of the wave function, $\phi_{n, x}(y)$, depends parametrically on the longitudinal coordinate $x$; the corresponding 'transverse' energy eigenvalue is $\varepsilon_{n, \perp}$ and is measured in units of the Fermi energy $E_{\mathrm{F}}$. Hence,

$$
\varepsilon_{n, \perp}=\left(\frac{\pi n}{k_{\mathrm{F}} d}\right)^{2}
$$

where $d$ is the width of the channel, for simplicity assumed to be constant.

The plus sign $(+)$ corresponds to propagation from the left to the right reservoir while the minus sign $(-)$ corresponds to transmission in the opposite direction. 
The dimensionless function $\Upsilon(x, t)$ is the total potential in units of $E_{F}$ felt by electrons moving in the electric field produced by the electrodes and the impurities in the doped region. We specify $\Upsilon$ as a sum $\Upsilon(x, t)=u_{0}(x)+\delta u(x, t)+v s(x)$ where $u_{0}(x) \approx e V_{g} / E_{\mathrm{F}}$ comes from the static part of the gate voltage. The fluctuating contribution from impurities and defects in the doped region is modeled by $\delta u(x, t)$, while $v$ is the potential caused by the source-drain voltage $\left(v=e V_{s d} / 2 E_{\mathrm{F}}\right)$. In order to match the Fermi levels in both reservoirs to the left and to the right, we must require that $s( \pm \infty)= \pm 1$. To simplify we have considered the case of small source-drain voltages and put $s(x)=0$. We also neglect the $x$-dependencies of the potentials $u_{0}$ and $\delta u$ giving

$$
\Upsilon(x, t)=u_{0}+\delta u(t) .
$$

For convenience we introduce a dimensionless parameter $\eta$,

$$
\left[n / \eta\left(u_{0}\right)\right]^{2} \equiv \varepsilon_{n, \perp}-u_{0}
$$

The use of the parameter $\eta$ allows for a particularly simple interpretation in terms of the well known conductance quantization with gate voltage [25]. If $\eta$ is an integer, say $m$, mode number $m$ is just about to start conducting. Small fluctuations in the gate and source-drain voltages may then induce variations in the conductance between $m-1$ and $m$ in units of the fundamental conductance quantum $2 e^{2} / h$. When $\eta=m+0.5$ we are exactly half way between two adjacent steps in the conductance when it is plotted versus gate voltage. From now on, $u_{0}$ is removed. The offset gate voltage $u_{0}$ which before affected the bottom of the potential well in which the electrons move, is now instead included in the effective width $d$ of the channel.

Note that according to our model the fluctuating part $\delta u$ is $x$-independent. The reason for making such an assumption is that in most ballistic structures the scattering potential is rather soft. This is so because the impurities are located rather far from the region occupied by the conducting electrons (see, e.g. Ref. [26]). Since we are interested in the role of an average potential, we may neglect the weak spatial dependence of $\delta u$. The noise properties in our model are thus entirely due to the dimensionless random quantity $\delta u(t)$. 
The general expression for the current in the absence of intermode scattering [1] is,

$$
I\left(V_{s d}, t\right)=\frac{2 e}{h} \int d E \delta n_{F}(E) \sum_{n} T_{n}[E, \delta u(t)]
$$

where $T_{n}[E, \delta u(t)]$ is the probability of transmission between the two reservoirs for an electron in mode $n$ having energy $E$. According to our simplified model, which will be used in the our numerical calculations, we assume that all the propagating modes have the same transparency $T$ at the Fermi level. Thus,

$$
T_{n}\left[E_{\mathrm{F}}, \delta u(t)\right]=\bar{T} \Theta\left[1-(n / \eta)^{2}-\delta u(t)\right]
$$

where $\Theta(x)$ is the Heaviside step function. The transmission probability is hence dependent on the quantity $\delta u(t)$. The current is driven by the difference in chemical potentials of the Fermi-Dirac distribution functions in the reservoirs,

$$
\delta n_{F}(E) \equiv n_{F}\left(E-E_{\mathrm{F}}-e V_{s d} / 2\right)-n_{F}\left(E-E_{\mathrm{F}}+e V_{s d} / 2\right) .
$$

(The total bias $V_{s d}$ is here chosen to be symmetrically shared by the distributions to the left and to the right.) We have studied the low temperature regime $(T=0)$ for small sourcedrain voltages, where this difference simplifies to $\delta n_{F}(E)=e V_{s d} \delta\left(E-E_{\mathrm{F}}\right)$. The current will in this case be,

$$
I\left(V_{s d}, t\right)=\frac{2 e^{2} V_{s d}}{h} \sum_{n} T_{n}\left[E_{\mathrm{F}}, \delta u(t)\right] .
$$

Since no scattering between the electronic states is taken into account, the only mechanism for changing the current is the possibility of having different numbers of propagating modes at different times.

\section{LOW-FREQUENCY NOISE}

\section{A. General consideration}

We consider fluctuations in $\delta u(t)$ as a random process composed of contributions from many elementary fluctuators. Each of them, let's say the $i$-th one, is described by a normalized random variable $\xi_{i}(t)$ changing between the two values \pm 1 with the corresponding 
transition rates $\Gamma_{i}^{+}$and $\Gamma_{i}^{-}$. Within the contact, this EF will create a potential of strength $a_{i}$ for the electrons. Transitions between the two different states are assumed to be induced by interactions with a thermal bath. The rates $\Gamma_{i}^{+}$and $\Gamma_{i}^{-}$are in general different and determined by the nature of hopping and the interaction between the EF and the thermal bath. From the detailed balance principle we find,

$$
\Gamma_{i}^{-} / \Gamma_{i}^{+}=\exp \left(-\Delta / k_{\mathrm{B}} T\right)
$$

where $T$ is the temperature and $\Delta$ the energy difference between the states of the EF. For high enough temperatures the rates are almost equal, while at low temperatures there will be a significant difference between the two. Here we consider the case $\Delta \ll k_{B} T \ll E_{\mathrm{F}}$, giving $\Gamma_{i}^{-}=\Gamma_{i}^{+} \equiv \Gamma_{i}$. The dependence of $\Gamma_{i}$ on $\Delta$ and $T$ is determined by the hopping mechanism (see discussion in Ref. [14]). If the transitions are due to quantum mechanical tunneling the rates are independent of temperature and we have $\Gamma_{i} \propto \Delta^{k}$. For EF-phonon interaction, $k=3$ while for EF-electron interaction, $k=1$. If transitions are induced by activation, $\Gamma_{i} \propto \exp \left(-W / k_{\mathrm{B}} T\right)$ where $W$ is some activation energy [14].

The number of fluctuators has been assumed to be large enough for substituting fixed values of the parameters for each fluctuator with values obtained from continuous distributions over $a$ and $\Gamma$. We assume these distributions to be uncorrelated, $\mathcal{P}(a, \Gamma) \equiv P_{a}(a) P_{\Gamma}(\Gamma)$. The nature of the statistical process will be completely defined by the distributions over $a$ and $\Gamma$ and the number of fluctuators $(\mathrm{N})$. Whether or not the noise in practice is caused by internal or external parameters is actually irrelevant. The result we have obtained applies to all cases when electrons in the channel experience a time dependent potential of the kind $\delta u(t)=\sum_{i=1}^{N} a_{i} \xi_{i}(t)$, where the distributions of the random quantities $a_{i}$ and $\Gamma_{i}$ are to be discussed below.

The gate potential $\delta u(t)$ (see Sec. II) is composed of the electrostatic potential from several EFs in the doping layer. As an approximation we may nevertheless assume the EFs to be uniformly distributed in a spherical shell $r_{\min } \leq r \leq r_{\max }$ ( $r$ being the radial coordinate measured from the center of the channel) in the doped region. The probability 
for finding an EF in a shell between $r$ and $r+d r$ is then $P(r) d r \propto r^{2} d r$. If we subtract the static background charge, we obtain a dipole potential, with a fluctuating sign from each EF (see e.g. Ref. [14]). This implies that the interaction parameter $a$ will be proportional to the inverse of the cube of the separation, $a \propto r^{-3}$. Normalizing under the assumption $a_{\max } / a_{\min } \gg 1$, we find the distribution of $P(a)$ to be

$$
P_{a}(a)=\frac{a_{\min }}{a^{2}}
$$

Even if the upper limit does not enter the expression for $P(a)$, it is important to include it in the following way. There must be an upper limit of possible fluctuations in the gate voltage since the number of fluctuators is finite. It will be given by $N a_{\max }$. For larger gate voltages the one- and two-point probabilities must be zero. But the approximation we have adopted to find these probabilities (see below) is only asymptotically $(N \rightarrow \infty)$ exact, and disregards this important fact. Therefore we have neglected the finite value of $a_{\max }$ throughout the derivation but included it at the end by truncating the two probability distributions over fluctuations in gate voltages. Note that while $a_{\text {min }}$ is related to the maximum distance to the impurities, $a_{\max }$ is related to the minimum distance.

The transition probabilities $\Gamma^{ \pm}$usually have an exponential dependence on the barrier parameters. Indeed, assume the EF to be described as an entity moving in a double well potential [22 24]. The logarithm of the transition probability is then proportional either to the barrier height (in the case of thermal activation), or to its strength (in the case of quantum tunneling). Since these heights have been found to have little spread among the different fluctuators, the distribution of $\ln (\Gamma)$ is smooth and almost uniform. We may hence model this distribution to be constant in the region between $\Gamma_{\min }$ and $\Gamma_{\max }$. As the result we obtain

$$
P_{\Gamma}(\Gamma)=\frac{1}{\ln \left(\Gamma_{\max } / \Gamma_{\min }\right)} \frac{1}{\Gamma},
$$

for the normalized distribution of $\Gamma$. 


\section{B. Analytical expression for noise intensity}

Noise is usually characterized by the current-current correlation function,

$$
S(\tau)=\langle I(t+\tau) I(t)\rangle_{t}-\langle I(t)\rangle_{t}^{2}
$$

or by its Fourier transform with respect to $\tau, S(\omega)$. The symbol $\langle\cdots\rangle_{t}$ means average over $t$ which is (under stationary conditions) the same as an ensemble average over the random process $\xi(t)$ (ergodicity).

From expression (10) for the current, we obtain the following current-current correlation function,

$$
\begin{aligned}
S(\tau)= & \left(\frac{2 e^{2} V_{s d}}{h}\right)^{2} \sum_{n, m}\left[\left\langle T_{n}(t) T_{m}(t+\tau)\right\rangle_{t}\right. \\
& \left.-\left\langle T_{n}(t)\right\rangle_{t}\left\langle T_{m}(t)\right\rangle_{t}\right] .
\end{aligned}
$$

The transmittance is $T_{n}(t) \equiv T_{n}\left[E_{\mathrm{F}}, \delta u(t)\right]$. In order to simplify the notation, we from now on substitute $\delta u$ with $u$. Since the gate voltage fluctuations must become uncorrelated for large time differences, expression (15) may be written as

$$
\begin{aligned}
S(\tau)= & \left(\frac{2 e^{2} V_{s d}}{h}\right)^{2} \sum_{n, m}[G(n, m \mid \tau)-G(n, m \mid \infty)] \\
G(n, m \mid \tau)= & \left\langle T_{n}(t) T_{m}(t+\tau)\right\rangle_{t} \\
& =\int d u \int d v T_{n}(u) T_{m}(v) P_{2}(u, v \mid \tau)
\end{aligned}
$$

The two-point probability of having gate voltage fluctuations $u$ and $v$ at times differing by $\tau$ is $P_{2}(u, v \mid \tau)$. $\quad P_{2}$ must split into one-point probabilities $P_{1}$ for large values of $\tau$, $\lim _{\tau \rightarrow \infty} P_{2}(u, v \mid \tau)=P_{1}(u) P_{1}(v)$.

The probabilities $P_{1}$ and $P_{2}$ may be calculated using a generating function,

$$
K_{N}(x, y)=\left\langle e^{-i x u(t)-i y v(t+\tau)}\right\rangle_{\xi_{i}},
$$

where $N$ denotes the number of fluctuators. The average is over all random quantities $\xi_{i}(t)$ representing elementary fluctuator $i$ with strength $a_{i}$ and transition rate $\Gamma_{i}$. From the definition of expectation values, 


$$
\begin{aligned}
& K_{N}(x, y \mid \tau)=\int e^{-i x u-i y v} P_{2}(u, v \mid \tau) d u d v, \\
& K_{N}(x, 0 \mid 0)=\int e^{-i x u} P_{1}(u) d u .
\end{aligned}
$$

These relations can easily be inverted to find $P_{1}$ and $P_{2}$ as functions of $K_{N}$,

$$
\begin{aligned}
P_{2}(u, v \mid \tau) & =\frac{1}{(2 \pi)^{2}} \int e^{i x u+i y v} K_{N}(x, y \mid \tau) d x d y, \\
P_{1}(u) & =\frac{1}{2 \pi} \int e^{i x u} K_{N}(x, 0 \mid 0) d x .
\end{aligned}
$$

Provided the elementary fluctuators are statistically independent, $K_{N}$ transforms into a product,

$$
K_{N}(x, y \mid \tau)=\prod_{i=1}^{N}\left\langle e^{-i x a_{i} \xi_{i}(t)-i y a_{i} \xi_{i}(t+\tau)}\right\rangle_{\xi_{i}} .
$$

Now consider factor number $i$ only. This is a strictly one-fluctuator quantity, and instead of fixing the values of the constants $a_{i}$ and $\Gamma_{i}$ we may introduce distribution functions over $a$ and $\Gamma$. Since then we have a function of these two parameters we have to perform a weighting over these distributions as well as the average over the statistical variable $\xi$,

$$
\left\langle K_{N}(x, y \mid \tau)\right\rangle_{e}=\left\langle K_{1}(x, y \mid \tau)\right\rangle_{e}^{N}
$$

where $\langle\cdots\rangle_{e}$ means an average over parameters $a, \Gamma$ describing the environment, while

$$
K_{1}(x, y \mid \tau)=\left\langle e^{-i x a \xi(t)-i y a \xi(t+\tau)}\right\rangle_{\xi}
$$

The last expression depends on the parameters $a$ and $\Gamma$. In order to evaluate $K_{N}$ we apply the Holtsmark procedure [27], which is applicable in the limit of many fluctuators,

$$
\begin{aligned}
& K_{N}(x, y \mid \tau)=e^{-F_{N}(x, y \mid \tau)} \\
& F_{N}(x, y \mid \tau)=N\left[1-\left\langle K_{1}(x, y \mid \tau)\right\rangle_{e}\right]
\end{aligned}
$$

This approximation is valid provided $1-K_{1}(x, y \mid \tau) \ll 1$ and $N \gg 1$. The generating function $K_{1}(x, y \mid \tau)$ for one fluctuator and fixed values of $a$ and $\Gamma$ has been calculated earlier 28] (see also Refs. [29,18]), 


$$
\begin{aligned}
K_{1}(x, y \mid \tau)= & e^{-\Gamma|\tau|}[\cosh (\Gamma|\tau|) \cos (a(x+y)) \\
& +\sinh (\Gamma|\tau|) \cos (a(x-y))]
\end{aligned}
$$

As is clearly seen, this expression decouples for large times into a product of functions of $x$ and $y$ as it must if gate voltage fluctuations at large time differences are uncorrelated. Equation (27) implies that $F_{N}$ in Eq. (26) will be given by

$$
\begin{aligned}
F_{N}(x, y \mid \tau) \equiv & -N\{\Phi(x+y)+\Phi(x-y) \\
& +\Psi(\tau)[\Phi(x+y)-\Phi(x-y)]\}
\end{aligned}
$$

The functions $\Phi$ and $\Psi$ are here defined as

$$
\begin{aligned}
\Phi(x) & =\frac{1}{2} \int_{a_{\min }}^{a_{\max }} P_{a}(a)[1-\cos (a x)] d a \\
& =\frac{a_{\min }}{2} \int_{a_{\min }}^{a_{\max }} \frac{1-\cos (a x)}{a^{2}} d a, \\
\Psi(\tau) & =\int_{\Gamma_{\min }}^{\Gamma_{\max }} P_{\Gamma}(\Gamma) e^{-2 \Gamma|\tau|} d \Gamma .
\end{aligned}
$$

Here we have made use of the distribution over fluctuator strengths (12). Using (13), $\Psi(\tau)$ can only be calculated analytically in the asymptotic limits,

$$
\Psi(\tau)=\left\{\begin{array}{cl}
1-\frac{2|\tau| \Gamma_{\max }}{\ln \left(\Gamma_{\max } / \Gamma_{\min }\right)}, & |\tau| \ll \Gamma_{\max }^{-1} \\
\frac{\ln \left(1 /|\tau| \Gamma_{\min }\right)}{\ln \left(\Gamma_{\max } / \Gamma_{\min }\right)}, & \Gamma_{\max }^{-1} \ll|\tau| \ll \Gamma_{\min }^{-1} \\
0, & |\tau| \gg \Gamma_{\min }^{-1}
\end{array}\right.
$$

The product $N a_{\text {min }}$, which in the following will be denoted as $4 C_{N} / \pi$, has the physical meaning of a typical shift of the QPC potential. We will show below that the typical value of $x$ in the function $\Phi(x)$ is $C_{N}^{-1}$. Hence, we replace the lower limit of integration in Eq. (29) by 0 . The behavior of $\Phi(x)$ depends on the relation between the upper limit $a_{\max }$ and $C_{N}$. We can also replace the upper limit by infinity provided,

$$
a_{\max } \gg C_{N}, \text { or } r_{\min } \ll \bar{r} \text {. }
$$

The constants $r_{\text {min }}$ and $\bar{r}$ are here the minimum and average distance, respectively, between the QPC and the EF. Taking the derivative of $\Phi$ twice, we obtain an integral representation of the Dirac delta function. Using its properties while integrating repeatedly we find, 


$$
\Phi(x)=\frac{\pi a_{\min }}{4}|x|, \quad a_{\max } x \gg 1
$$

In the opposite limiting case,

$$
\Phi(x)=\frac{a_{\min } a_{\max }}{4} x^{2}, \quad a_{\max } x \ll 1 .
$$

Under the conditions (32) $F_{N}$ in Eq. 26 is found to be

$$
\begin{aligned}
F_{N}(x, y, \tau)= & -C_{N}[(1+\Psi(\tau))|x+y| \\
& +(1-\Psi(\tau))|x-y|] .
\end{aligned}
$$

The Fourier transform with respect to $x$ and $y$ needed to get $P_{2}$, given by Eq. (21), is readily found from (26) and (35) to be a product of two Lorentzian functions. Similarly, from (22) we calculate $P_{1}$ to be a simple Lorentzian function. Unfortunately, because of the slow decay of the Lorentzians, our approximations made so far are not sufficient for calculating the noise. We must also take into account that for large values of the fluctuations $\left(u, v \geq N a_{\max }\right)$, the approximation (35) breaks down. We correct for this by introducing truncation factors $\mathcal{D}$ for each variable representing fluctuations in the gate voltage in the probabilities,

$$
\begin{aligned}
P_{1}(u) & =\mathcal{D}_{1}(u) \mathcal{L}\left(u \mid 2 C_{N}\right), \\
P_{2}(u, v \mid \Psi) & =\frac{1}{2} \mathcal{D}_{2}(u) \mathcal{D}_{2}(v) \mathcal{L}_{+} \mathcal{L}_{-}, \\
\mathcal{L}_{+} & =\mathcal{L}\left(\frac{u+v}{2} \mid C_{N}(1+\Psi)\right), \\
\mathcal{L}_{-} & =\mathcal{L}\left(\frac{u-v}{2} \mid C_{N}(1-\Psi)\right), \\
\mathcal{D}_{i}(u) & =\frac{\Theta\left(N a_{\max }-|u|\right)}{\mathcal{N}_{i}} \\
\mathcal{L}(z, w) & =\frac{1}{\pi} \frac{w}{z^{2}+w^{2}} .
\end{aligned}
$$

The function $\Psi(\tau)$ is given by (30), and has the asymptotic values 1 for $t \rightarrow 0$ and 0 for $t \rightarrow$ $\infty$. The necessary renormalization factor imposed by the truncation procedure is $\mathcal{N}_{i}$. For $P_{2}$ this constant has to be calculated numerically, but for $P_{1}, \mathcal{N}_{1}=2 \arctan \left(N a_{\max } / \pi\right)$. Note that such a truncation has an obvious physical meaning - the fluctuation of, say, $u$ cannot 
be greater than $N a_{\max }$. This shift represents the case when all fluctuator contributions add up coherently to maximal total potential felt by the electrons.

The final expression for the current-current correlation function will be

$$
\begin{aligned}
S(\tau)= & \left(\frac{2 e^{2} V_{s d}}{h}\right)^{2} \sum_{n, m} \int_{-\infty}^{w_{n}} d u \int_{-\infty}^{w_{m}} d v \\
& \times\left[P_{2}(u, v \mid \Psi(\tau))-P_{2}(u, v \mid 0)\right] .
\end{aligned}
$$

The upper limits of integration are here given by $w_{n} \equiv 1-(n / \eta)^{2}$, and $P_{2}$ by (37) assuming $\bar{T}=1$. Finally, the noise spectrum is found as the Fourier transform of $S(\tau)$.

Let us compare our result with the conventional formalism for calculating noise in electronic systems. To be general, assume the current to be dependent on a random quantity $u$ that fluctuates in time around the average value $u_{0}=\langle u\rangle_{t}$. In our case it will be the effective gate voltage but the nature of this quantity is not of crucial importance here. As a random variable, $u$ possesses certain one- and two-point probabilities $P_{1}(u)$ and $P_{2}(u, v \mid \tau)$, similar to what was presented in the preceding section. Further, consider the case for which the current is a smooth function of $u$ and typical fluctuations are small. Then we are allowed to expand the current as a function of the variable $u$,

$$
I[u(t)]=I\left(u_{0}\right)+G\left(u_{0}\right) \delta u(t)+\mathcal{O}\left\{[u(t)]^{2}\right\}
$$

where the transconductance $G$ is defined as $G\left(u_{0}\right)=\partial I / \partial u \|_{u=u_{o}}$. Note that in our case this should not at all be an adequate procedure - the transmission probability being one for propagating modes and zero for reflected ones is certainly not a differentiable function of the gate voltage. Nevertheless we may compare the two results to see if they deviate significantly. The expansion Eq. (43) directly implies that an approximate expression for the current-current correlation function can be written down as

$$
\begin{aligned}
S(\tau)= & \langle I(t+\tau) I(t)\rangle_{t}-\langle I(t)\rangle_{t}^{2} \\
& \approx G^{2}\left(u_{0}\right)\langle\delta u(t+\tau) \delta u(t)\rangle_{t}
\end{aligned}
$$

In this approach the correlation function factorizes into two parts. One describes the rigid 
part of the system and the other is solely determined by the time-dependent random quantities. As previously, substitute $\delta u$ with $u$ to simplify the notation.

In our study, $u$ is the fluctuating part of the gate voltage and $G$ the transconductance. The latter can be evaluated as the derivative of the average current with respect to the gate voltage. From (10) and (36) we easily find the average current to be,

$$
\begin{aligned}
\langle I(t)\rangle_{t} & =\frac{2 e^{2} V_{s d} T}{h \mathcal{N}} \sum_{k=1}\left[\frac{1}{\pi} \arctan \left(\frac{u_{k}}{2 C_{N}}\right)+\mathcal{N} / 2\right], \\
u_{k} & =\min \left\{1-\left(\frac{k}{\eta}\right)^{2}, N a_{\max }\right\},
\end{aligned}
$$

where the sum is over propagating modes. The transconductance will then be,

$$
G(\eta)=-\frac{2 e^{2} V_{s d} T}{h \mathcal{N}} \sum_{k=k_{\min }}^{k_{\max }} \mathcal{L}\left(1-\left(\frac{k}{\eta}\right)^{2} \mid 2 C_{N}\right) .
$$

Here, $k_{\min }=\left[1+\eta \sqrt{1-N a_{\max }}\right]$ and $k_{\max }=\left[\eta \sqrt{1+N a_{\max }} ;[\cdots]\right.$ denotes the integer part. $\eta$ is related to $u_{0}$ according to (7). The average $\langle u(t+\tau) u(t)\rangle_{t}$ can be evaluated when the truncation is done in the variables $(u \pm v) / 2$ instead of $u$ and $v$. This modification is not less accurate than the truncation itself. Making use of (37) but with this modified truncation we find,

$$
\begin{aligned}
& \langle u(t+\tau) u(t)\rangle_{t}=-4 \Psi C_{N}^{2} \\
& +\left(N a_{\max }\right)^{2}\left[\mathcal{A}\left(\frac{B}{1+\Psi(\tau)}\right)-\mathcal{A}\left(\frac{B}{1-\Psi(\tau)}\right)\right], \\
& \mathcal{A}(x)=1 / x \arctan (x), \quad B=4 a_{\max } / \pi a_{\min }
\end{aligned}
$$

where $\Psi(\tau)$ is given by (30). If $N a_{\max } \gg \pi C_{N}$ (which is also required for using the Holtsmark approximation [27]), the obvious restriction $\lim _{\tau \rightarrow 0}\langle u(t+\tau) u(t)\rangle_{t}>0$ is fulfilled. Furthermore, since $\langle u(t)\rangle_{t}=0$ we must have $\lim _{\tau \rightarrow \infty}\langle u(t+\tau) u(t)\rangle_{t}=\langle u(t+\tau)\rangle_{t}\langle u(t)\rangle_{t}=0$, where we have assumed that fluctuations in the effective gate voltage become uncorrelated at large time differences. Obviously, (48) obeys this condition. Collecting Eqs. (44), (47) and (48) we may compare this conventional approach of evaluating noise to our calculation. In Fig. (2) we indeed find, as expected, significant deviations between the two treatments. At the sec- 
ond conductance step, they differ approximately by a factor 25 . It should be emphasized that this comparison is strongly dependent on the choice of the parameter $N a_{\text {min }}$.

The case opposite to (32) for which $a_{\max } \geq a_{\min }$, is applicable to a structure with a very wide spacer compared to the thickness of the doping layer. The Lorentzians $\mathcal{L}(z \mid w)$ in Eq. (36) - (37) are then replaced by Gaussians,

$$
\mathcal{G}(z \mid w)=\frac{1}{w \sqrt{2 \pi}} e^{-z^{2} / 2 w^{2}}
$$

and the quantity $C_{N}$ by $N \sqrt{a_{\min } a_{\max }}$ [see Eq. (34)]. However, we do not further pursue the analysis of this situation.

\section{Numerical analysis}

We had to resort to a numerical calculation of the noise using a discrete cosine transform.

In general there is a problem with discrete transforms that is called aliasing [30]. It occurs when the time sequence is not bandwidth limited, and shows up as an increased Fourier transform for high frequencies. In short, high frequency components outside the range where the transform can be calculated are folded into this domain. For the case of investigating $1 / f$-like noise this problem is severe. The contribution to the transform that is transferred into the region of interest would even diverge if the transform was exactly proportional to $1 / f$. When the signal has a finite bandwidth this problem does not occur. To invent an artificial bandwidth in our case we decrease the physical parameter $\Gamma_{\max }$ to be of the same order as the upper limit of the Fourier transform of $S(\tau)$. Evidently, this will be allowed since the numerically calculated transform anyway will be cut in the vicinity of the upper limit.

If the function of time can only be sampled in a relatively few number of points because of computational difficulties there is also a problem to find the transform over a wide range of frequencies. Basically, it is only possible to find the transform over a number of decades of the order $\left[\log _{10}\right.$ (number of sample points) - 1]. We solve this problem in the following way. 
The time scale is set by the upper limit of $\tau, \tau_{\max }$. By changing its value we may successively map out different parts of the Fourier transform over a wide range of frequencies. For low frequencies $\left(\omega<100 \Gamma_{\min }\right)$, a large value of $\tau_{\max }$ is used and $S(\tau)$ is sampled over almost the entire domain.

For high frequencies though, only the most rapidly varying part of $S(\tau)$ is relevant because the contribution from the remaining parts is averaged out when the transform is calculated. Hence it is sufficient to select the most rapid variations, which in our case are close to $\tau=0$. Keeping the same number of sample points while $\tau_{\max }$ is decreased, the resolution in time is enhanced. With an increased resolution we are able to compute the high frequency components. Note also that when changing $\tau_{\max }$ we must change $\Gamma_{\max }$ accordingly in the way mentioned above. Unfortunately, because we don't sample $S(\tau)$ for all values of $\tau$ two problems occur. Firstly, the calculated transform will have an offset. We correct this by adjusting the transform to the low frequency solution where $\tau_{\max }$ is very large. Secondly, there will be rapid oscillations in the transform because we truncate $S(\tau)$ with a sharp step when $\tau_{\max }$ is decreased. This problem is solved by filtering, averaging over a small interval yields the correct form of the transform. In practice we convolve the Fourier transform using a constant filtering function with sufficiently large bandwidth (in time). (Note that time and frequency are exchanged here compared to the 'normal' case when we filter a function of time.) We repeat the calculation and decrease $\tau_{\max }$ in sufficiently small steps (we used a factor of 10) every time. Finally, we put the pieces together and match each new section at the lowest frequency. In this way we are able to find the transform over a wide frequency range. Under any circumstances, the transform for $\omega<100 \Gamma_{\min }$ is not affected at all by our method of calculation. A smooth matching (see Fig. 3) of the pieces provides some numerical evidence that the method is adequate.

The parameter $\left(N a_{\mathrm{min}} / E_{\mathrm{F}}\right)$ describing a typical fluctuation in the potential from all impurities was chosen to be equal to $5 \cdot 10^{-3}$; the maximal fluctuation was determined by $a_{\max } / a_{\min }=100$. For the Holtsmark approximation [27] to be valid a large ratio between the maximal and average potential fluctuation is required, $a_{\max } / N a_{\min } \gg 1$ (see also the end of 
subsection [IIB]). With these parameters we may for instance have 10 impurities implying $a_{\max } / N a_{\min }=10$, which seems plausible. As has already been mentioned, the transmission probability $T_{n}(u)$ was taken to be zero for non conducting modes and unity for conducting ones. In this case we will find that $\sum_{n=1}^{\infty} T_{n}(u)=[\eta]$, where $[\eta]$ denotes the integer part of $\eta$, which gives the number of conducting modes at zero fluctuation in gate voltage.

A comparison of our calculation with the simple-minded conventional approach leading to (44) is done in Fig. 2. Large deviations are found for this set of parameters, at the step $S_{B}(\omega) / S_{A}(\omega) \approx 25$. Indeed, significant deviations are expected due to the non-differentiable character of the transmittance as a function of gate voltage. The step-like shape of the average transmittance is responsible for the strongly enhanced level at the step and the very low level of noise on the plateaus.

In Fig. 3, the logarithm of the noise is plotted in units of $\left(2 e^{2} V_{s d} / h\right)^{2}$ versus the logarithm of dimensionless frequency $w=\omega / \Gamma_{\min }$. The graphs show the noise at the second conductance step $(\eta=2)$ as well as between the second and third step $(\eta=2.5)$. Generally, the current fluctuations are maximal when $\eta$ is an integer. This corresponds to a step in the current-gate voltage curve. Since noise is just the Fourier transform of the current-current correlation function, we consequently expect the noise to be large for integer and small for non integer values of $\eta$. This is also clearly seen in Fig. 3 .

If $N a_{\max } \leq E_{\mathrm{F}} /[\eta]$ noise is actually independent of $a_{\min }$ at the step. This can be understood in the following way. The two Lorentzian functions in the two point probability (37) is in this case split symmetrically into two regions of positive and negative gate voltage fluctuations. These two domains are defined by $T_{n}$ being constant. The tails of the probability distribution only extend into the nearest plateau on both sides of the step for this value of $a_{\text {max }}$. It is then irrelevant how wide the Lorentzians are (determined by $N a_{\min } / E_{\mathrm{F}}$ ), as long as the total weight of probability remains the same in the different domains. Only the probability density profile differ for different values of $N a_{\min } / E_{\mathrm{F}}$. But since $T_{n}$ is constant in both regions, it has no effect on $S$. By measuring the level of noise at and between the steps we may infer the order of magnitude of the parameter $a_{\min }$. It will be related to the ratio of 
the two noise intensities provided $N a_{\max } \leq E_{\mathrm{F}} /[\eta]$. For the presented results in Fig. [3, we actually have $N a_{\max }=E_{\mathrm{F}} /[\eta]$.

Between the steps in the current-gate voltage characteristics the noise must decrease with $a_{\mathrm{min}}$, since the average potential fluctuations are proportional to this parameter. This has also been checked.

From Fig. 3 we deduce that in all cases, the dependence is almost linear for frequencies larger than some typical value $\omega_{c}$. It is determined by the parameters $\eta$ and $N a_{\text {min }} / E_{\mathrm{F}}$. For $\eta=2$ corresponding to the second step in conductance quantization, $\omega_{c} \approx 2 \Gamma_{\text {min }}$ irrespective

of the value of $N a_{\min } / E_{\mathrm{F}}$ when this quotient is sufficiently small $\left(<1 \cdot 10^{-3}\right)$. If the system is biased between the second and third step $\eta=2.5, \omega_{c}$ is of the order $10 \Gamma_{\min }$. This threshold frequency has been found to slightly increase with decreasing $N a_{\text {min }}$ between the steps. Because of computational difficulties, the form of the curves for, let's say $\omega>10^{4} \Gamma_{\min }$, should only be considered to be accurate within a relative error of the order $5 \%$. The slopes for $\omega>\omega_{c}$ were found to be close to $-0.85(-0.93)$ for $\eta=2.0(\eta=2.5), S(\omega) \propto 1 / \omega^{0.85(0.93)}$. This means that the noise is almost $1 / f$-like.

Experimentally, one is also interested in the integrated noise intensity per decade. For the curves in Fig. 3, this quantity is shown in Fig. 1. The integration is for decade number $k$ performed over the interval $[k-0.5, k+0.5]$. The center frequency is here $\omega_{c}$, which in turn defines $k, k=\omega_{c} / \Gamma_{\min }$. If the noise was identically $1 / f$, the integrated noise intensity per decade would be constant for all decades. In reality, the noise must be cut for frequencies larger than the physical value of $\Gamma_{\max }$. In realistic systems $\Gamma_{\max }$ can be as large as $10^{12} \Gamma_{\min }$. This is beyond the limits of our calculation, so we are unable to detect this physical cut-off.

\section{DISCUSSION AND CONCLUSIONS}

We would like to stress that the calculated level of noise significantly differed from a conventional estimate, see Fig. 2. (It is lower by a factor 25 for one set of parameters). The approximation was based upon an expansion of the current-current correlation function in 
fluctuations of the gate voltage. Due to the sharp structure of the average transmittance $\left\langle T_{n}\right\rangle_{t}$ the conventional approach leads to invalid results. The deviation from the true result depends on the strength of the fluctuators - the weaker they are the larger is the difference.

According to our model the current-current correlation function $S(\tau)$ (42) has the following properties. Under certain restrictions (see Appendix A) it is independent of the number of propagating modes, $[\eta]$. Furthermore, if the spacer is thick enough and the inequality $N a_{\max }<E_{\mathrm{F}} /[\eta]$ holds, $S(\tau)$ is also independent of $a_{\min }$ at the steps in conductance quantization. Hence, measuring the noise level at a step would be enough to find the value of $a_{\max }$ which is the interaction strength between the nearest elementary fluctuator and the QPC.

Our model (which assumes a constant transmittance $T_{n}$ on every plateau) suggests that the level of noise is very low close to the middle of a plateau. Generally, it is more dependent on the maximum $\left(r_{\max } \propto 1 / a_{\min }\right)$ than the minimum $\left(r_{\min } \propto 1 / a_{\max }\right)$ distance to the EFs. This fact provides us with a way of determining $a_{\min }$ almost independently of $a_{\text {max }}$. Since $a_{\text {min }}$ then effectively is the only parameter, the level of noise at a plateau where many modes are conducting should provide sufficient information to determine its value. Having obtained both parameters of the model one can examine its validity by comparing the predicted and measured dependence of the noise on the gate voltage.

Numerical calculations indicate that there is a typical frequency, $\omega_{c}$, specific for each set of parameters and above which $S(\omega) \propto 1 / \omega^{\alpha}$. The exponent $\alpha$ was found to be slightly less than 1 and the threshold frequency $\omega_{c}$ close to the minimal transition rate $\Gamma_{\min }$ for the fluctuators. Also, on the plateaus $\alpha$ is slightly enhanced compared to its value at the steps. Further, $\omega_{c}$ increased when the minimum fluctuator strength $a_{\min }$ decreased.

Experiments on $1 / f$-noise in QPC [31] are in qualitative agreement with our theoretical results. Indeed, the noise was found to be $S(\omega) \propto I^{\beta} / \omega^{\alpha}$, where $\beta=2 \pm 0.4$ and $\alpha=$ $0.9 \pm 0.1$. As we also conclude, the value of $\alpha$ was larger on the plateaus than at the steps in conductance quantization with gate voltage. Of course, the level of the noise was larger at the steps than between them. For the highest two values of the number of conducting modes, 4 and 5, the noise was almost the same in agreement with our claims (see appendix 
A). It was also pointed out that after some cool-downs of the sample the peaks of the noise as a function of the parameter $\eta$ were smeared out. As a possible cause for this effect we suggest that the elementary fluctuators may change their positions between subsequent measurements because of heating. This would affect $a_{\min }$ as well as $a_{\max }$ and give rise to the observed effect.

Finally, we recall that our derivation of noise is done in the limit of many fluctuators, $N \gg 1$. At the same time, we assume that $N \ll\left(r_{\max } / r_{\min }\right)^{2}$. The last inequality means that a significant number of EFs are situated in a close vicinity of the QPC. The probability to have a large time-dependent fluctuation of the electric potential is large and governed by a Lorentzian distribution function (37). If the last inequality does not hold only the nearest $\mathrm{EF}$ is of importance and the distribution is a Gaussian function. If $N$ is of order 1 , the noise has a random telegraph character.

\section{ACKNOWLEDGMENTS}

This work was supported by NorFA, grant no. 93.30 .155 and by the Nordic Research Network on the Physics of Nanometer Electronic Devices. JPH gratefully acknowledges the hospitality of the Department of Physics at Oslo University, Norway. We would like to thank Mats Jonson, Alexander Zagoskin and Robert Shekhter for proofreading the manuscript.

\section{APPENDIX A: NOISE FOR DIFFERENT NUMBERS OF PROPAGATING MODES}

The magnitude of the noise will here be shown to be independent, under certain restrictions, of the number of propagating modes through the contact. Let zero fluctuation in gate voltage correspond to a fixed position on the quantized plateaus in the currentgate voltage characteristics. Assume that the Lorentzians in the two-point probability

extend over $p$ steps in the $I-V_{g}$ curve. This provides a restriction on the value of $\eta$, $\eta \geq p$. We have then $T_{n}(u)=1$ for all modes $n \leq[\eta]-p \equiv q$ and gate voltages $u$ 
and $v$ for which $P_{2}(u, v \mid \Psi(\tau))=P_{2}(u, v \mid \tau)$ is finite. The dimensionless correlation function $s(\tau) \equiv\left(h / 2 e^{2} V_{s d}\right)^{2} S(\tau)$ (see 42$)$ can then be reduced,

$$
s(\tau)=\sum_{n, m=q+1} \int d u \int d v T_{m}(u) T_{n}(v) \mathcal{P}(u, v \mid \tau)
$$

where $\mathcal{P}(u, v \mid \tau)=P_{2}(u, v \mid \tau)-P_{2}(u, v \mid 0)$. Indeed, for terms with both $T$ s constant, $T_{n}=$ $T_{m} \equiv 1$ the integral $\int d u \int d v \mathcal{P}(u, v \mid \tau)$ vanishes because of the normalization condition. On the other hand, if only one $T$ is constant, let's say $T_{m} \equiv 1$ the integrals $\int d u \mathcal{P}(u, v \mid \tau)$ vanish because

$$
\int d u P_{2}(u, v \mid \tau)=P_{1}(v)
$$

is $\tau$-independent. Note that $T_{n}$ is dependent of the offset gate voltage which is related to $\eta$. Because of the simple form of $T_{n}$, being zero for reflected modes and one for propagating modes, we are able to make a translation in the mode index,

$$
T_{n}(u, \eta)=T_{n-q}(u, \eta-q)
$$

Finally, changing indices of summation we obtain,

$$
\begin{aligned}
s(\tau)= & \sum_{\substack{n^{\prime}, m^{\prime}=1\\
}} \int d u \int d v \\
& \times T_{m^{\prime}}(u,\{\eta\}+p) T_{n^{\prime}}(v,\{\eta\}+p) \mathcal{P}(u, v \mid \tau)
\end{aligned}
$$

where $\{\eta\} \equiv \eta-[\eta]$ is the fractional part of $\eta$. Evidently, this is independent of the number of propagating modes, $[\eta]$. 


\section{REFERENCES}

[1] C. W. J. Beenakker and H. van Houten, Solid State Physics 44, 1 (1991).

[2] B. J. van Wees, H. van Houten, . G. Williamson, L. P. Kouwenhoven, D. van der Marel, and C. T. Foxon, Phys. Rev. Lett. 60, 848 (1988).

[3] L. P. Kouwenhoven, B. J. van Wees, C. J. P. M. Harmans, J. G. Williamson, H. van Houten, C. W. J. Beenakker, C. T. Foxon, and J. J. Harris, Phys. Rev. B 39, 8040 (1989).

[4] D. A. Wharam, T. J. Thornton, R. Newbury, M. Pepper, H. Ahmed, J. E. F. Frost, D. G. Hasko, D. C. Peacock, d. A. Ritetae, and G. A. C. Jones, J. Phys. C 21, L209 (1988).

[5] L. I. Glazman and A. V. Khaetskii, Europhys. Lett. 9, 263 (1989).

[6] A. M. Zagoskin, Pis'ma Zh. Eksp. Tepr. Fiz. 52, 1043 (1990) [JETP Lett. 52, 435 (1991)].

[7] N. K. Patel, J. T. Nichols, L. Martin-Moreno, M. Pepper, J. E. F. Frost, D. A. Ritchie, and G. A. C. Jones, Phys. Rev. B 44,13 549 (1991).

[8] K. S. Ralls, W. J. Skocpol, L. D. Jackel, R. E. Howard, L. A. Fetter, R. W. Epworth and D. M. Tennant, Phys. Rev. Lett. 52, 228 (1984).

[9] C. T. Rogers and R. A. Buhrman, Phys. Rev. Lett. 53, 1272 (1984).

[10] K. S. Ralls and R. A. Buhrman, Phys. Rev. Lett. 60, 2434 (1988).

[11] D. C. Ralph and R. A. Buhrman, Phys. Rev. Lett. 69, 2118 (1992).

[12] N. M. Zimmerman, B. Golding, and W. H. Haemmerle, Phys. Rev. Lett. 67, 1322 (1991).

[13] B. Golding, N. Zimmerman, and S. N. Coppersmith, Phys. Rev. Lett. 68, 998 (1992). 
[14] Yu. M. Galperin, V. G. Karpov, and V. I. Kozub, Advances in Physics 38, 669 (1989).

[15] S. Hershfeld, J. H. Davies, and J. W. Wilkins, Phys. Rev. Lett. 67, 3720 (1991).

[16] Y. Meir, N. S. Wingreen, and P. A. Lee, Phys. Rev. Lett. 66, 3048 (1991); 70, 2601 (1993).

[17] A. Levi Yeyati, A. Martin-Rodero, and F. Flores, Phys. Rev. Lett. 71, 2991 (1993).

[18] J. P. Hessling and Yu. M. Galperin, Göteborg APR 94-38, cond-mat/9408081 (Submitted to Phys. Rev. B. 25 Aug. 1994).

[19] P. E. Lindelof, private communication.

[20] R. Taboryski, A. K. Geim, and P. E. Lindeløf, Superlattices and Microstructures 12, 137 (1992).

[21] R. Taboryski, A. K. Geim, M. Persson, and P. E. Lindeløf, Phys. Rev. B. 49, 7813 (1994).

[22] P. W. Anderson, B. I. Halperin, and C. M. Varma, Philos. Mag. 25, 1 (1972).

[23] W.A. Phillips, J. Low Temp. Phys. 7, 351 (1972).

[24] V. G. Karpov, M. I. Klinger, and F. N. Ignat'ev, Solid State Commun. 44, 333 (1982); Sov. Phys. JETP 57, 439 (1983).

[25] L. I. Glazman, G. B. Lesovik, D. E. Khmelnitskii, and R. I. Shekhter, Pis'ma Zh. Eksp. Teor. Fiz. 48, 218 (1988) [JETP Lett. 48, 238 (1988)].

[26] T. Ando, A. B. Fowler, and F. Stern, Rev. Mod. Phys. 54, 437 (1982).

[27] S. Chandrasekhar, Rev. Mod. Phys. 15, 1 (1943).

[28] S. M. Rytov, Introduction to the Statistical Radiophysics (Nauka, Moscow 1976).

[29] Yu. M. Galperin, V. L. Gurevich, V. I. Kozub, Sov. Phys. Solid State 31, 807 (1989). 
[30] W. H. Press, B. P. Flannery, S. A. Teukolsky, W. T. Vetterling Numerical Recipes (Cambridge University Press 1990).

[31] Yuan P. Li, D. C. Tsui, J. J. Heremans, J. A. Simmons and G. W. Weimann, Appl. Phys. Lett. 57 (8), 774 (1990). 


\section{FIGURES}

FIG. 1. A quantum point contact is defined at the interface between the undoped AlGaAs spacer layer and GaAs by the split gate confinement technique. Noise is caused by the motion of impurities in the n-doped AlGaAs layer.

FIG. 2. Logarithm of noise, $\log _{10}\left[S\left(\omega=100 \Gamma_{\min }\right)\right]$ as a function of $\eta$. The unit of $S(\omega)$ is $\left(2 e^{2} V_{s d} T / h\right)^{2}$. The parameter $\eta$ gives the operating point, i.e. where on the conductance-gate voltage staircase the system is centered. The integer part of $\eta$ is the number of conducting modes. We calculated $S_{a}$ as the numerical Fourier transform of the valid expression for the noise, Eq. (42). Using the traditional approach, $S_{b}$ was computed from Eq. (44, 47-49). The minimum fluctuator potential $a_{\min }$ was defined by $N a_{\min } / E_{\mathrm{F}}=5 \times 10^{-3}\left(E_{\mathrm{F}}\right.$ being the Fermi energy); $a_{\max }$ by $a_{\max } / a_{\min }=100$.

FIG. 3. Logarithm of noise, $\log _{10}[S(\omega)]$ (in units of $\left(2 e^{2} V_{s d} T / h\right)^{2}$ ) as a function of the logarithm of dimensionless frequency, $\log _{10}(w)\left(\omega=w \Gamma_{\min }\right)$. The minimum fluctuator potential $a_{\min }$ was defined by $N a_{\min } / E_{\mathrm{F}}=5 \times 10^{-3}\left(E_{\mathrm{F}}\right.$ being the Fermi energy); $a_{\max }$ by $a_{\max } / a_{\min }=100$. The calculation was done for two different values of $\eta, \eta=2$ where mode number 2 is just about to become propagating, and $\eta=2.5$. The numbers indicate the approximate slope $k\left(S(\omega) \propto \omega^{k}\right)$ at frequency $\omega=100 \Gamma_{\min }$.

FIG. 4. Integrated noise intensity per decade as a function of dimensionless center frequency $w_{c}=\omega_{c} / \Gamma_{\min }$. The different plots correspond to the same cases as in Fig. $3, \eta=2$, and $\eta=2.5$. The integration was performed over the interval $w \in\left[w_{c}-0.5, w_{c}+0.5\right]$. If $S(\omega) \propto 1 / \omega^{\alpha}$, where $\alpha=1$, the bars would be of equal height. 